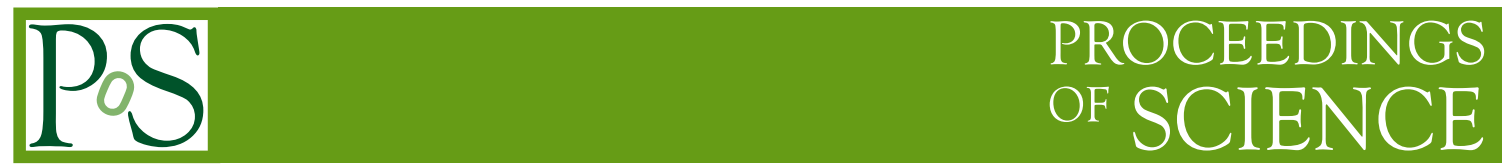

\title{
Theoretical Aspects of the Flavour Sector
}

\author{
Wolfgang Altmannshofer ${ }^{a, *}$ \\ ${ }^{a}$ Department of Physics and Santa Cruz Institute for Particle Physics, \\ University of California, Santa Cruz, CA 95064, USA \\ E-mail: waltmann@ucsc.edu
}

In this talk I give an overview of recent theoretical developments in flavor physics. I cover the determination of the CKM matrix, I discuss the status of the $B$ anomalies, and I summarize exciting news on rare Kaon decays.

The Eighth Annual Conference on Large Hadron Collider Physics-LHCP2020

25-30 May, 2020

online

${ }^{*}$ Speaker 


\section{Introduction}

Flavor is a puzzling aspect of particle physics. Indeed, many of the open questions in particle physics are related to flavor, for example: Why is the spectrum of the Standard Model (SM) quarks and leptons so hierarchical? Does the Higgs boson give mass to all SM fermions? Are there new sources of flavor and CP violation beyond the SM? Are the current flavor anomalies hints for new physics (NP)? Is flavor a portal to dark sectors.

In this talk, I will briefly discuss a few developments in the field of flavor physics. In section 2, I will summarize the status of our knowledge of the CKM matrix, highlighting the impact of a recent improvement in the Standard Model prediction of the observable $\epsilon_{K}$. In section 3, I will give a status report on the so-called $B$ anomalies that persist in the latest experimental updates by LHCb, and discuss possible new physics implications. Finally, in section 4, I will share the latest news concerning the search for the rare Kaon decays $K \rightarrow \pi v \bar{v}$ and their possible connection to dark sectors.

\section{Developments in the Determination of the CKM Matrix}

In the SM, all flavor changing processes are determined by the Cabibbo-Kobayashi-Maskawa (CKM) matrix. The CKM matrix can be described by the four Wolfenstein parameters $\lambda, A, \bar{\rho}$, and $\bar{\eta}$ that can be defined in terms of CKM entries in the following way

$$
\lambda^{2}=\frac{\left|V_{u s}\right|^{2}}{\left|V_{u s}\right|^{2}+\left|V_{u d}\right|^{2}}, \quad A^{2} \lambda^{2}=\frac{\left|V_{c b}\right|^{2}}{\left|V_{u s}\right|^{2}+\left|V_{u d}\right|^{2}}, \quad \bar{\rho}+i \bar{\eta}=-\frac{V_{u d} V_{u b}^{*}}{V_{c d} V_{c b}^{*}} .
$$

In the SM, these parameters can be overconstrained by a large set of experimental measurements, including the rates of leptonic and semi-leptonic decays of Kaons, D-mesons, B-mesons, and Bbaryons, Kaon and B-meson oscillation parameters, as well as CP-violating asymmetries in B-meson decays. The global fits that are performed show overall good consistency $[1,2]$ and indicate that the $\mathrm{CKM}$ picture of flavor and CP violation holds with high accuracy. Long-standing tensions at the $2 \sigma-3 \sigma$ level exist between the inclusive and exclusive determinations of the CKM elements $V_{c b}$ and $V_{u b}$ [3], and recently a tension in first row CKM unitarity might have re-emerged [4-6]. While these tensions require continued attention, there is little doubt that all observed flavor violating processes that enter the CKM fits are dominated by SM physics, with NP contributions constrained at the level of $O(10 \%)$.

The latest CKM fit results in the context of the SM read [7]

$$
\lambda=0.22484_{-0.00006}^{+0.00025}, \quad A=0.823_{-0.014}^{+0.005}, \quad \bar{\rho}=0.157_{-0.006}^{+0.010}, \quad \bar{\eta}=0.350_{-0.007}^{+0.008} .
$$

The observable $\epsilon_{K}$ that measures indirect CP violation in Kaon mixing is an important input to the CKM fits. Precise theory predictions for $\epsilon_{K}$, however, turn out to be challenging as the perturbative expansion of the charm quark contribution seems to converge very slowly [8]. Recently, it was found that a clever rearrangement of the perturbative series leads to a significant improvement of the convergence behavior [9]. With the new treatment, the perturbative uncertainty in $\epsilon_{K}$ is now subdominant, resulting in a significantly increased constraining power of $\epsilon_{K}$ in CKM fits. The main impact is a reduction of uncertainty of the Wolfenstein parameter $A$. With the old theory treatment 
one finds $A=0.824_{-0.017}^{+0.006}$ [7], which has to be compared to the more precise $A=0.823_{-0.014}^{+0.005}$ from eq. (2).

\section{Status of the B Anomalies}

For several years there exist persistent discrepancies between SM predictions and experimental

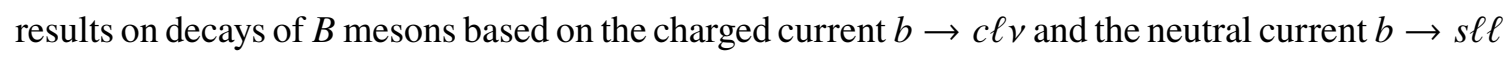
transitions. Particularly interesting are the hints for lepton flavor universality (LFU) violation, as the measured ratios of branching fractions

$$
R_{D^{(*)}}=\frac{\mathrm{BR}\left(B \rightarrow D^{(*)} \tau v\right)}{\mathrm{BR}\left(B \rightarrow D^{(*)} \ell v\right)}, \quad R_{K^{(*)}}=\frac{\mathrm{BR}\left(B \rightarrow K^{(*)} \mu^{+} \mu^{-}\right)}{\mathrm{BR}\left(B \rightarrow K^{(*)} e^{+} e^{-}\right)},
$$

are under excellent theoretical control.

Starting with LFU violation in the neutral current decays, the most precise measurements of $R_{K}$ and $R_{K^{*}}$ to date are from $\mathrm{LHCb}[10,11]$ and read

$$
\begin{gathered}
R_{K}=0.846_{-0.054-0.014}^{+0.060+0.016}, \quad \text { for } 1.1 \mathrm{GeV}^{2}<q^{2}<6 \mathrm{GeV}^{2}, \\
R_{K^{*}}= \begin{cases}0.66_{-0.07}^{+0.11} \pm 0.03, & \text { for } 0.045 \mathrm{GeV}^{2}<q^{2}<1.1 \mathrm{GeV}^{2}, \\
0.69_{-0.07}^{+0.11} \pm 0.05, & \text { for } 1.1 \mathrm{GeV}^{2}<q^{2}<6 \mathrm{GeV}^{2},\end{cases}
\end{gathered}
$$

with $q^{2}$ being the dilepton invariant mass squared. The SM predicts $R_{K}^{\mathrm{SM}} \simeq R_{K^{*}}^{\mathrm{SM}} \simeq 1$ [12] with uncertainties that are much smaller than the experimental ones. The experimental results are approximately $2.5 \sigma$ below the SM prediction for each of the three measurements.

A recent result on lepton flavor universality in baryonic decays $\Lambda_{b} \rightarrow p K \ell \ell$ [13]

$$
R_{p K}=0.86_{-0.11}^{+0.14} \pm 0.05, \quad \text { for } 0.1 \mathrm{GeV}^{2}<q^{2}<6 \mathrm{GeV}^{2}
$$

points in the same direction. However, given its large uncertainty it is also compatible with the SM expectation $R_{p K}^{\mathrm{SM}} \simeq 1$.

The first step in discussing the NP implications of the apparent discrepancies consists of a model independent effective Hamiltonian analysis, with NP parameterized in terms of Wilson coefficients of dimension 6 operators. The experimental results on $R_{K}$ and $R_{K^{*}}$ can be accommodated by either suppressing the decay modes into muons or enhancing the decay modes into electrons. It was found that the operators that can consistently accomplish this are

$$
\begin{aligned}
\mathcal{H}_{\mathrm{eff}}=\mathcal{H}_{\mathrm{eff}}^{\mathrm{SM}} & -\frac{4 G_{F}}{\sqrt{2}} V_{t b} V_{t s}^{*} \frac{e^{2}}{16 \pi^{2}}\left(C_{9}^{b s e e}\left(\bar{s} \gamma_{\alpha} P_{L} b\right)\left(\bar{e} \gamma^{\alpha} e\right)+C_{10}^{b s e e}\left(\bar{s} \gamma_{\alpha} P_{L} b\right)\left(\bar{e} \gamma^{\alpha} \gamma_{5} e\right)\right. \\
+ & \left.C_{9}^{b s \mu \mu}\left(\bar{s} \gamma_{\alpha} P_{L} b\right)\left(\bar{\mu} \gamma^{\alpha} \mu\right)+C_{10}^{b s \mu \mu}\left(\bar{s} \gamma_{\alpha} P_{L} b\right)\left(\bar{\mu} \gamma^{\alpha} \gamma_{5} \mu\right)\right) .
\end{aligned}
$$

Recent studies that discuss the above operators in view of the latest $R_{K}$ and $R_{K^{*}}$ measurements as well as a multitude of additional experimental data on rare $B$ decays can be found in [14-19].

Focusing on the muonic operators and their Wilson coefficients $C_{9}^{b s \mu \mu}$ and $C_{10}^{b s \mu \mu}$, the parameter space that fits the measured $R_{K}$ and $R_{K^{*}}$ best is shown in blue in the left plot of Fig. 1. Intriguingly, the region of parameter space that is preferred by the LFU observables is fully compatible with 

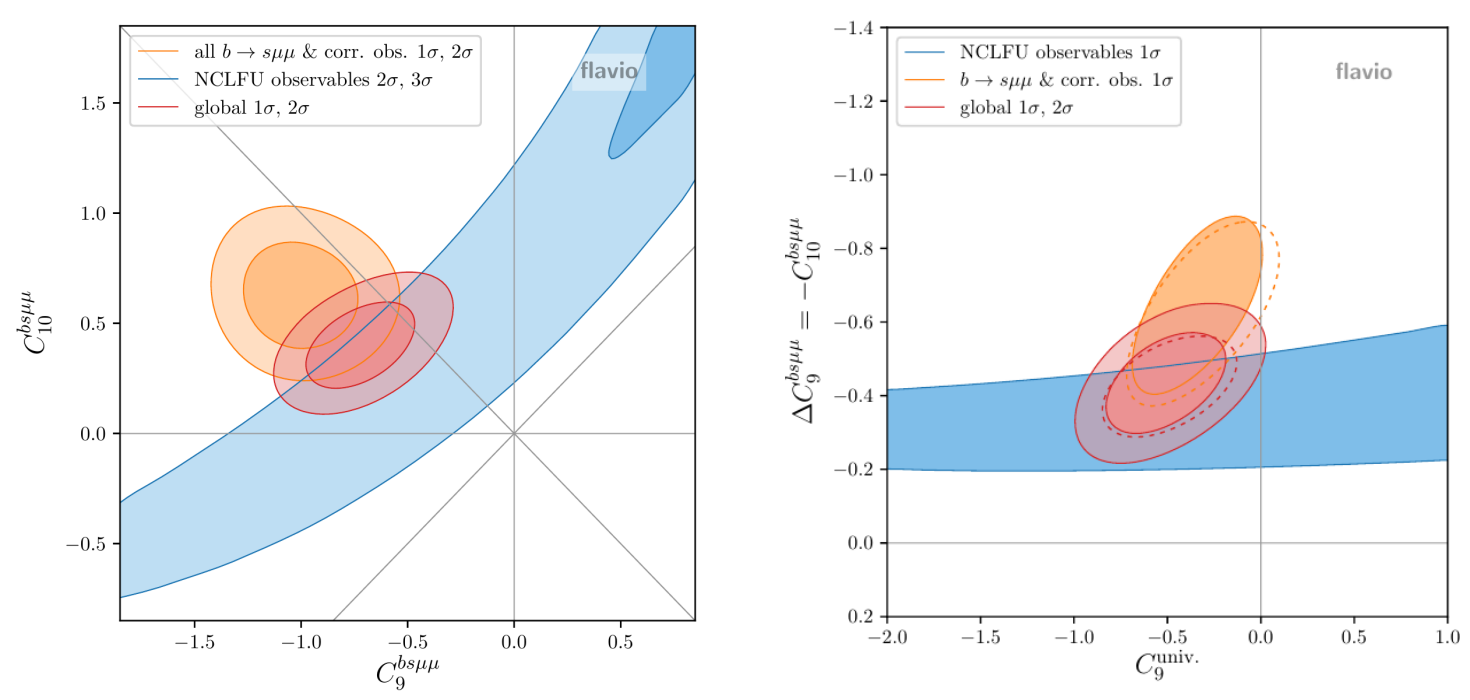

Figure 1: Preferred regions in the plane of the Wilson coefficients $C_{9}^{b s \mu \mu}$ and $C_{10}^{b s \mu \mu}$ (left), and $C_{9}^{\text {univ. and }}$ $C_{9}^{b s \mu \mu}=-C_{10}^{b s \mu \mu}$ (right) at the scale of the $b$ quark mass. The plots are updates from [14] including the latest LHCb results on $B \rightarrow K^{*} \mu \mu$ [20] and are based on the global likelihood in Wilson coefficient space provided by smelli [22], built on flavio [23] and wilson [24], see [25].

other anomalies that are seen in the muonic decays $B \rightarrow K^{*} \mu \mu, B \rightarrow K \mu \mu, B_{s} \rightarrow \phi \mu \mu$, and $B_{s} \rightarrow \mu \mu$ (orange region). Those include, for example, the discrepancy in the angular distribution of the decay products in $B \rightarrow K^{*} \mu \mu$ (parameterized in particular by the observable $P_{5}^{\prime}$ ) [20], and the $B_{s} \rightarrow \phi \mu \mu$ decay which is observed at a rate significantly lower than the SM predicts [21].

Overall, excellent fits can be obtained in 1 parameter NP scenarios with either a muonic vector current $C_{9}^{b s \mu \mu}$ by itself, or with a left-handed muonic current $C_{9}^{b s \mu \mu}=-C_{10}^{b s \mu \mu}$. In global fits, the precise pull values of these scenarios depend on the assumptions about hadronic uncertainties. Taking into account only the theoretically robust LFU ratios, pull values of $\sim 4 \sigma$ are found. Even better fits can be obtained by adding also a lepton flavor universal interaction $C_{9}^{\text {univ. }}\left(\bar{s} \gamma_{\nu} P_{L} b\right) \sum_{\ell}\left(\bar{\ell} \gamma^{\nu} \ell\right)$ with a best fit point $C_{9}^{b s \mu \mu}=-C_{10}^{b s \mu \mu} \simeq C_{9}^{\text {univ. }} \simeq-0.45$, as shown in the right plot of Fig. 1 .

The best fit values for the Wilson coefficients $C_{i}$ can be translated into a scale of NP, $\Lambda_{\mathrm{NP}}$, via

$$
\frac{1}{\Lambda_{\mathrm{NP}}^{2}}=\frac{4 G_{F}}{\sqrt{2}} \frac{e^{2}}{16 \pi^{2}}\left|V_{t s}^{*} V_{t b}\right|\left|C_{i}\right| \simeq \frac{\left|C_{i}\right|}{(35 \mathrm{TeV})^{2}} .
$$

A simple NP model that is able to explain the $b \rightarrow s \ell \ell$ data is an extension of the SM by a $Z^{\prime}$ gauge boson based on gauging the difference of muon and tau number, $L_{\mu}-L_{\tau}[26,27]$. The $L_{\mu}-L_{\tau}$ gauge symmetry leads to vectorial couplings of the $Z^{\prime}$ to muons (but not electrons) and can give the preferred value for $C_{9}^{b s \mu \mu}$ for a broad range of $Z^{\prime}$ masses from tens of $\mathrm{GeV}$ to several $\mathrm{TeV}$. It should be noted that Ref. [26] aimed at explaining the $P_{5}^{\prime}$ anomaly and predicted the violation of LFU in $b \rightarrow s \ell \ell$ decays at the $\sim 25 \%$ level, in agreement with the subsequent experimental results on $R_{K}$ and $R_{K^{*}}$. Additional characteristic predictions of the $L_{\mu}-L_{\tau}$ model are slightly enhanced rates of $b \rightarrow s \tau \tau$ decays (by $\sim 25 \%$ ), SM-like rates of $B_{s} \rightarrow \mu \mu$ and $b \rightarrow s v v$ decays, and no lepton flavor violating decays of $b$ hadrons. Recently, the $L_{\mu}-L_{\tau}$ model has been extended to include also flavor universal axial vector currents [28]. 
Turning to the case of the charged current decays, the experimental world averages of $R_{D}$ and $R_{D^{*}}$ from the heavy flavor averaging group (HFLAV) read [29]

$$
R_{D}=0.340 \pm 0.027 \pm 0.013, \quad R_{D^{*}}=0.295 \pm 0.011 \pm 0.008,
$$

with an error correlation of $\rho=-38 \%$. The corresponding SM predictions are known with high precision [30-32]. The values adopted by HFLAV are [29]

$$
R_{D}^{\mathrm{SM}}=0.299 \pm 0.003, \quad R_{D^{*}}^{\mathrm{SM}}=0.258 \pm 0.005 .
$$

The combined discrepancy between the SM prediction and experimental world averages of $R_{D}$ and $R_{D^{*}}$ is quoted as 3.1 $\sigma$. Model independent NP analyses find that the best description of all the available $b \rightarrow c \tau v$ data is obtained by rescaling the SM operator $\left(\bar{c} \gamma_{\alpha} P_{L} b\right)\left(\bar{\tau} \gamma^{\alpha} P_{L} v\right)$ [33-36]. The corresponding NP scale is found to be a few TeV.

Interestingly enough, it is possible to find combined explanations of all the discussed $B$ anomalies, i.e. both the neutral current and charged current anomalies. In a model independent approach based on the SM effective field theory (SMEFT), one finds that two NP couplings are sufficient to simultaneously explain $R_{D}, R_{D^{*}}, R_{K}, R_{K^{*}}, P_{5}^{\prime}$, and the suppressed $b \rightarrow s \mu \mu$ branching ratios. The operators point to leptoquarks with masses of at most a few TeV. Such leptoquarks could be the remnant of an extended gauge sector, e.g. so-called 4321 models, or (Pati-Salam) ${ }^{3}$ models [37, 38]. Attempts have also been made to give explanations of the $B$ anomalies in the context of supersymmetric models with R-parity violation [39-43]. Predictions of most scenarios that attempt a combined explanation are order of magnitude enhanced rates for the rare decays $B_{s} \rightarrow \tau \tau, B \rightarrow K^{(*)} \tau \tau$ and often also lepton flavor violating decays like $B_{s} \rightarrow \tau \mu, B \rightarrow K^{(*)} \tau \mu$, possibly in reach of $\mathrm{LHCb}$.

\section{News on Rare Kaon Decays}

The rare Kaon decays $K \rightarrow \pi v \bar{v}$ are highly sensitive probes of NP. Their branching ratios are extremely small and can be predicted with high accuracy in the SM [44, 45]

$$
\mathrm{BR}\left(K^{+} \rightarrow \pi^{+} v \bar{v}\right)_{\mathrm{SM}}=(8.4 \pm 1.0) \times 10^{-11}, \quad \mathrm{BR}\left(K_{L} \rightarrow \pi^{0} v \bar{v}\right)_{\mathrm{SM}}=(3.4 \pm 0.6) \times 10^{-11} .
$$

The charged and neutral decay modes are related, and in very generic classes of SM extensions one finds the so-called Grossman-Nir (GN) bound [46]

$$
\mathrm{BR}\left(K_{L} \rightarrow \pi^{0} v \bar{v}\right) \lesssim 4.3 \times \mathrm{BR}\left(K^{+} \rightarrow \pi^{+} v \bar{v}\right) .
$$

The $K^{+} \rightarrow \pi^{+} v \bar{v}$ decay is currently searched for at the NA62 experiment. At the time of the LHCP 2020 conference, few $K^{+} \rightarrow \pi^{+} \nu \bar{v}$ events had been observed at NA62, corresponding to a branching ratio of [47]

$$
\mathrm{BR}\left(K^{+} \rightarrow \pi^{+} v \bar{v}\right)_{\mathrm{NA} 62}=\left(4.7_{-4.7}^{+7.9}\right) \times 10^{-11} .
$$

The neutral counterpart, $K_{L} \rightarrow \pi^{0} v \bar{v}$, is searched for at the KOTO experiment. Interestingly, in a 2019 analysis, KOTO saw few events in the signal region, with only $\sim 0.1$ events expected [48]. An unofficial interpretation in terms of a $K_{L} \rightarrow \pi^{0} v \bar{v}$ signal found [49]

$$
\operatorname{BR}\left(K_{L} \rightarrow \pi^{0} v \bar{v}\right) \approx\left(210_{-110}^{+200}\right) \times 10^{-11}
$$


This branching ratio is not only two orders of magnitude above the SM prediction, but also exceeds the GN bound by a factor of few.

In fact, in the presence of light new states, the GN bound can be violated. One simple example is given by the following scenario [50]: Introduce a light, invisible, and sufficiently long-lived NP particle, $X$, with couplings that give rise to the decay $K \rightarrow \pi X$. As neither $X$ nor neutrinos are detected in the NA62 and KOTO experiments, such a decay mode can mimic the $K \rightarrow \pi v \bar{v}$ signature. However, if the mass of $X$ is close to the pion mass, the NA62 experiment would not be able to detect the exotic $K^{+} \rightarrow \pi^{+} X$ decay, due to the overwhelming SM background from $K^{+} \rightarrow \pi^{+} \pi^{0}$. On the other hand, the KOTO experiment retains its sensitivity for $m_{X} \sim m_{\pi}$. Due to the "blind spot" of NA62, such a scenario can seemingly violate the GN bound.

The events seen at KOTO invited many speculations about their possible NP origin [49, 51-62]. Many viable NP scenarios have been identified, methods to distinguish exotic Kaon decays with light, invisible states from the $K \rightarrow \pi v \bar{v}$ decays have been suggested, and the unique role of NA62 and KOTO to probe dark sector physics has been emphasized.

Note added: At the ICHEP 2020 conference, new results by NA62 and KOTO have been presented. NA62 finds a $3.5 \sigma$ evidence for $K^{+} \rightarrow \pi^{+} \nu \bar{v}$ compatible with the SM prediction [63]

$$
\mathrm{BR}\left(K^{+} \rightarrow \pi^{+} v \bar{v}\right)_{\mathrm{NA} 62}=\left(11.0_{-3.5}^{+4.0} \pm 0.3\right) \times 10^{-11},
$$

The KOTO collaboration has revised its background estimate, quoting an expected number of $1.05 \pm 0.28$ background events [64]. This reduces the significance of the observed events. An official KOTO interpretation of the observed events in terms of a $K_{L} \rightarrow \pi^{0} v \bar{v}$ branching ratio is expected soon.

\section{Conclusions}

The $B$ anomalies endure. Model independent effective Hamiltonian analyses show that there exist remarkably economic new physics explanations in the form of four fermion contact interactions with generic new physics scales of the order of few tens of TeV (in the case of $R_{K}$ and $R_{K^{*}}$ ) or few $\mathrm{TeV}$ (in the case of $R_{D}$ and $R_{D^{*}}$ ). The leading new physics models contain either $Z^{\prime}$ gauge bosons (in the case of $R_{K}$ and $R_{K^{*}}$ ) or leptoquarks (in the case of $R_{D}$ and $R_{D^{*}}$ and combined explanations).

Rare Kaon decays are once more taking on a prominent role in the search for new physics. The NA62 experiment is on track for a discovery and subsequent precision measurement of the $K^{+} \rightarrow \pi^{+} v \bar{v}$ decay. Searching for $K_{L} \rightarrow \pi^{0} v \bar{v}$, the KOTO experiment recently observed few events far above the SM expectation. Interpreted as an actual signal, such a Kaon anomaly would have spectacular implications pointing to light dark sector particles being produced in Kaon decays.

While it remains to be seen if any of the current anomalies in flavor physics will survive further experimental and theoretical scrutiny, there is no doubt that low energy flavor probes will remain crucial tools to explore physics beyond the Standard Model for the foreseeable future.

\section{Acknowledgements}

WA would like to thank the organizers of the LHCP 2020 conference for the invitation to give this presentation and Peter Stangl for the updated plots in Fig. 1. The research of WA was supported by the National Science Foundation under Grant No. PHY-1912719. 


\section{References}

[1] J. Charles et al. [CKMfitter Group], Eur. Phys. J. C 41, no.1, 1-131 (2005) [arXiv:hep$\mathrm{ph} / 0406184$ [hep-ph]], updated results and plots available at: http://ckmfitter .in2p3. fr

[2] M. Bona et al. [UTfit], JHEP 10, 081 (2006) [arXiv:hep-ph/0606167 [hep-ph]], updated results and plots available at: http://www.utfit.org/UTfit/

[3] P. A. Zyla et al. [Particle Data Group], PTEP 2020, no.8, 083 C01 (2020).

[4] C. Y. Seng, M. Gorchtein, H. H. Patel and M. J. Ramsey-Musolf, Phys. Rev. Lett. 121, no.24, 241804 (2018) [arXiv:1807.10197 [hep-ph]].

[5] B. Belfatto, R. Beradze and Z. Berezhiani, Eur. Phys. J. C 80, no.2, 149 (2020) [arXiv:1906.02714 [hep-ph]].

[6] A. Czarnecki, W. J. Marciano and A. Sirlin, Phys. Rev. D 100, no.7, 073008 (2019) [arXiv:1907.06737 [hep-ph]].

[7] S. Descotes-Genon, talk at the LHC Results Forum, May 8, 2020.

[8] J. Brod and M. Gorbahn, Phys. Rev. Lett. 108, 121801 (2012) [arXiv:1108.2036 [hep-ph]].

[9] J. Brod, M. Gorbahn and E. Stamou, [arXiv:1911.06822 [hep-ph]].

[10] R. Aaij et al. [LHCb], Phys. Rev. Lett. 122 (2019) no.19, 191801 [arXiv:1903.09252 [hep-ex]].

[11] R. Aaij et al. [LHCb], JHEP 08 (2017), 055 [arXiv:1705.05802 [hep-ex]].

[12] M. Bordone, G. Isidori and A. Pattori, Eur. Phys. J. C 76 (2016) no.8, 440 [arXiv:1605.07633 [hep-ph]].

[13] R. Aaij et al. [LHCb], JHEP 05, 040 (2020) [arXiv:1912.08139 [hep-ex]].

[14] J. Aebischer, W. Altmannshofer, D. Guadagnoli, M. Reboud, P. Stangl and D. M. Straub, Eur. Phys. J. C 80 (2020) no.3, 252 [arXiv:1903.10434 [hep-ph]].

[15] M. Alguero, B. Capdevila, A. Crivellin, S. Descotes-Genon, P. Masjuan, J. Matias, M. Novoa Brunet and J. Virto, Eur. Phys. J. C 79 (2019) no.8, 714 [arXiv:1903.09578 [hep-ph]].

[16] M. Ciuchini, A. M. Coutinho, M. Fedele, E. Franco, A. Paul, L. Silvestrini and M. Valli, Eur. Phys. J. C 79 (2019) no.8, 719 [arXiv:1903.09632 [hep-ph]].

[17] K. Kowalska, D. Kumar and E. M. Sessolo, Eur. Phys. J. C 79 (2019) no.10, 840 [arXiv:1903.10932 [hep-ph]].

[18] A. Datta, J. Kumar and D. London, Phys. Lett. B 797 (2019), 134858 [arXiv:1903.10086 [hep-ph]].

[19] A. Arbey, T. Hurth, F. Mahmoudi, D. M. Santos and S. Neshatpour, Phys. Rev. D 100 (2019) no.1, 015045 [arXiv:1904.08399 [hep-ph]]. 
[20] R. Aaij et al. [LHCb], Phys. Rev. Lett. 125, no.1, 011802 (2020) [arXiv:2003.04831 [hep-ex]].

[21] R. Aaij et al. [LHCb], JHEP 09, 179 (2015) [arXiv:1506.08777 [hep-ex]].

[22] J. Aebischer, J. Kumar, P. Stangl and D. M. Straub, Eur. Phys. J. C 79, no.6, 509 (2019) [arXiv: 1810.07698 [hep-ph]].

[23] D. M. Straub, [arXiv:1810.08132 [hep-ph]].

[24] J. Aebischer, J. Kumar and D. M. Straub, Eur. Phys. J. C 78, no.12, 1026 (2018) [arXiv:1804.05033 [hep-ph]].

[25] P. Stangl, talk at the IPPP workshop "Beyond the Flavour Anomalies", April 1, 2020.

[26] W. Altmannshofer, S. Gori, M. Pospelov and I. Yavin, Phys. Rev. D 89, 095033 (2014) [arXiv:1403.1269 [hep-ph]].

[27] W. Altmannshofer and I. Yavin, Phys. Rev. D 92, no.7, 075022 (2015) [arXiv:1508.07009 [hep-ph]].

[28] W. Altmannshofer, J. Davighi and M. Nardecchia, Phys. Rev. D 101, no.1, 015004 (2020) [arXiv:1909.02021 [hep-ph]].

[29] Y. S. Amhis et al. [HFLAV], [arXiv:1909.12524 [hep-ex]], and online updates at https://hflav.web.cern.ch/

[30] F. U. Bernlochner, Z. Ligeti, M. Papucci and D. J. Robinson, Phys. Rev. D 95 (2017) no.11, 115008 [arXiv: 1703.05330 [hep-ph]].

[31] D. Bigi, P. Gambino and S. Schacht, JHEP 11 (2017), 061 [arXiv:1707.09509 [hep-ph]].

[32] S. Jaiswal, S. Nandi and S. K. Patra, JHEP 12 (2017), 060 [arXiv:1707.09977 [hep-ph]].

[33] C. Murgui, A. Peñuelas, M. Jung and A. Pich, JHEP 09, 103 (2019) [arXiv:1904.09311 [hep-ph]].

[34] P. Asadi and D. Shih, Phys. Rev. D 100, no.11, 115013 (2019) [arXiv:1905.03311 [hep-ph]].

[35] R. X. Shi, L. S. Geng, B. Grinstein, S. Jäger and J. Martin Camalich, JHEP 12, 065 (2019) [arXiv:1905.08498 [hep-ph]].

[36] K. Cheung, Z. R. Huang, H. D. Li, C. D. Lü, Y. N. Mao and R. Y. Tang, [arXiv:2002.07272 [hep-ph]].

[37] L. Di Luzio, A. Greljo and M. Nardecchia, Phys. Rev. D 96, no.11, 115011 (2017) [arXiv:1708.08450 [hep-ph]].

[38] M. Bordone, C. Cornella, J. Fuentes-Martin and G. Isidori, Phys. Lett. B 779, 317-323 (2018) [arXiv:1712.01368 [hep-ph]]. 
[39] N. G. Deshpande and X. G. He, Eur. Phys. J. C 77, no.2, 134 (2017) [arXiv:1608.04817 [hep-ph]].

[40] W. Altmannshofer, P. S. Bhupal Dev and A. Soni, Phys. Rev. D 96, no.9, 095010 (2017) [arXiv:1704.06659 [hep-ph]].

[41] K. Earl and T. Gregoire, JHEP 08, 201 (2018) [arXiv:1806.01343 [hep-ph]].

[42] S. Trifinopoulos, Eur. Phys. J. C 78, no.10, 803 (2018) [arXiv:1807.01638 [hep-ph]].

[43] W. Altmannshofer, P. S. B. Dev, A. Soni and Y. Sui, Phys. Rev. D 102, no.1, 015031 (2020) [arXiv:2002.12910 [hep-ph]].

[44] J. Brod, M. Gorbahn and E. Stamou, Phys. Rev. D 83, 034030 (2011) [arXiv:1009.0947 [hep-ph]].

[45] A. J. Buras, D. Buttazzo, J. Girrbach-Noe and R. Knegjens, JHEP 11, 033 (2015) [arXiv:1503.02693 [hep-ph]].

[46] Y. Grossman and Y. Nir, Phys. Lett. B 398, 163-168 (1997) [arXiv:hep-ph/9701313 [hep-ph]].

[47] G. Ruggiero, talk at the KAON 2019 conference.

[48] S. Shinohara, talk at the KAON 2019 conference.

[49] T. Kitahara, T. Okui, G. Perez, Y. Soreq and K. Tobioka, Phys. Rev. Lett. 124, no.7, 071801 (2020) [arXiv:1909.11111 [hep-ph]].

[50] K. Fuyuto, W. S. Hou and M. Kohda, Phys. Rev. Lett. 114, 171802 (2015) [arXiv:1412.4397 [hep-ph]].

[51] D. Egana-Ugrinovic, S. Homiller and P. Meade, Phys. Rev. Lett. 124, no.19, 191801 (2020) [arXiv:1911.10203 [hep-ph]].

[52] P. S. B. Dev, R. N. Mohapatra and Y. Zhang, Phys. Rev. D 101, no.7, 075014 (2020) [arXiv:1911.12334 [hep-ph]].

[53] Y. Jho, S. M. Lee, S. C. Park, Y. Park and P. Y. Tseng, JHEP 04, 086 (2020) [arXiv:2001.06572 [hep-ph]].

[54] J. Liu, N. McGinnis, C. E. M. Wagner and X. P. Wang, JHEP 04, 197 (2020) [arXiv:2001.06522 [hep-ph]].

[55] X. G. He, X. D. Ma, J. Tandean and G. Valencia, JHEP 04, 057 (2020) [arXiv:2002.05467 [hep-ph]].

[56] R. Ziegler, J. Zupan and R. Zwicky, JHEP 07, 229 (2020) [arXiv:2005.00451 [hep-ph]].

[57] Y. Liao, H. L. Wang, C. Y. Yao and J. Zhang, [arXiv:2005.00753 [hep-ph]].

[58] S. Gori, G. Perez and K. Tobioka, [arXiv:2005.05170 [hep-ph]]. 
[59] M. Hostert, K. Kaneta and M. Pospelov, [arXiv:2005.07102 [hep-ph]].

[60] A. Datta, S. Kamali and D. Marfatia, Phys. Lett. B 807, 135579 (2020) [arXiv:2005.08920 [hep-ph]].

[61] J. Aebischer, A. J. Buras and J. Kumar, [arXiv:2006.01138 [hep-ph]].

[62] W. Altmannshofer, B. V. Lehmann and S. Profumo, [arXiv:2006.05064 [hep-ph]].

[63] R. Marchevski, talk at ICHEP 2020, July 28, 2020.

[64] N. Shimizu, talk at ICHEP 2020, July 28, 2020. 